Research Paper

\title{
Landscape of FGF/FGFR Alterations in 12,372 Chinese Cancer Patients
}

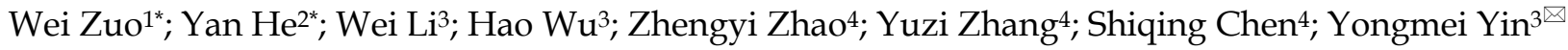 \\ 1. Department of Respiratory, The First Affiliated Hospital of Nanchang University, Jiangxi, China \\ 2. The Second Shenzhen People's hospital, The First Affiliated Hospital of Shenzhen University, Shenzhen, China \\ 3. Department of Oncology, The First Affiliated Hospital of Nanjing Medical University, Nanjing, China \\ 4. The Medical Department, 3D Medicines, Inc. Shanghai, China \\ *Dr. Wei Zuo and Yan He contributed equally to this work and serve as co-first authors. \\ $\square$ Corresponding author: Dr. Yongmei Yin, MD, PhD. Department of Oncology, The First Affiliated Hospital of Nanjing Medical University. NO.300 \\ Guangzhou Road. Nanjing 210029, China. Tel: 86-13951842727; Email: ym.yin@hotmail.com
}

(c) The author(s). This is an open access article distributed under the terms of the Creative Commons Attribution License (https://creativecommons.org/licenses/by/4.0/). See http://ivyspring.com/terms for full terms and conditions.

Received: 2020.06.09; Accepted: 2020.08.18; Published: 2020.09.23

\begin{abstract}
Purpose: The aberrant of fibroblast growth factors and their receptors (FGF/FGFR) is an emerging target in the treatment of solid tumors. This study aimed to explore the landscape of FGF/FGFR alterations in a large cohort of cancer patients.

Material and Methods: The formalin-fixed paraffin-embedded specimens of cancer patients who have underwent next-generation sequencing (NGS) from 2017 to 2019 in 3DMed Clinical Laboratory Inc. were included in this study.

Findings: Of 12,372 Chinese cancer patients with more than 20 tumor types $(60 \%$ male, median age, 58.0 [IQR, 49.0-66.0]), genomic alterations in FGF, FGFR, and both were observed in 895 (7.2\%), 862 (7.0\%), and $186(1.5 \%)$ patients, respectively. The highest prevalence of FGF/FGFR mutations fell in esophagus cancer $(61.6 \%, 98 / 159)$ and urinary tract cancer $(52.7 \%, 145 / 275)$. The most common pathway-level mutations were FGFR single nucleotide variants $(635,5.1 \%)$ and FGF amplifications $(628$, $5.1 \%)$. The microsatellite instability status was negatively associated with amplifications $(p=0.0017)$.

Conclusion: FGF/FGFR alterations were widely occurred in cancer patients, and the mutational landscape may contribute to the further study design and development of FGF/FGFR inhibitors.
\end{abstract}

Key words: Fibroblast growth factor receptor; Next-generation sequencing; Solid tumor

\section{Introduction}

Fibroblast growth factor (FGF) receptors (FGFRs) are highly conserved transmembrane tyrosine kinase receptors (RTKs), which are crucial in a variety of physiological process, such as the regulation of development, differentiation, survival, and migration of cells. Upon the binding of FGF, FGFR kinases will be dimerized and autophosphorylated, thus activating multiple transduction pathways, including the Ras/mitogen-activated protein kinase (MAPK) pathway, the canonical and non-canonical Wnt pathway, and the phosphatidylinositol-4,5bisphosphate 3-kinase (PI3K)-AKT pathway, which all play importantly roles in the cancer biological process [1-3]. The crosstalk between FGF/FGFR with the oncogenic pathways also provide a rationale for the therapeutic strategy for cancer treatment.

In tumor cells, aberrant FGFR signaling can be resulted from the amplification, fusion or missense mutations of FGF or FGFR family members, which are considered as promising targets for cancer treatment $[4,5]$. In addition, FGF/FGFR signaling has been reported to be mediate resistance to oncotherapy including chemotherapy, radiotherapy and targeted therapy $[6,7]$. Agents that target FGF/FGFR axis were shown to inhibit angiogenesis and sometimes reverse the acquired resistance of oncotherapy in various cancers [8-10]. Currently, more than one hundred clinical trials are ongoing in a variety of advanced 
cancers with aberrant FGF/FGFR signaling, including several basket trials in solid tumors. Currently, FDA has approved four multikinase inhibitors with FGFR as one of the targets, including ponatinib, regorafenib, pazopanib, and lenvatinib. In April 2019, the first pan-FGFR inhibitor erdafitinib was approved by FDA for the later-line treatment of locally advanced or metastatic urothelial carcinoma with FGFR2 or FGFR3 genetic alterations based on an ORR of $32.2 \%$ from BLC2001 study, a phase 2 trial (NCT02365597) [11, 12]. Most recently on April 17, 2020, pemigatinib, an selective inhibitor of FGFR isoforms 1, 2 and 3 got approval for FGFR2+ cholangiocarcinoma later line treatment upon the data from the FIGHT-202 study with an ORR of $36 \%$ [13]. In the present study, we aimed to investigate the spectrum of FGF/FGFR alterations in a large cohort of Chinese cancer patients through next-generation sequencing to aid the future study design and development of FGF/FGFR-inhibiting drugs.

\section{Methods}

\section{Clinical specimens}

The Formalin-Fixed Paraffin-Embedded (FFPE) specimens of solid tumor patients who have underwent next-generation sequencing (NGS) from 2017 to 2019 in 3DMed Clinical Laboratory Inc. were included. The diagnosis of the specimens was confirmed by hematoxylin and eosin (H\&E) staining by an independent pathologist. The specimens were required to have a percentage of tumor cells over $20 \%$ and a size $\geq 1 \mathrm{~mm}$ for further analysis.

\section{NGS sequencing}

DNAs extracted from the FFPE tumor specimens were sequenced in NGS platform with a well-designed 381 cancer gene panel on Illumina Nextseq 500 to $>500 \mathrm{X}$ coverage as previously described[14] in 3DMed Clinical Laboratory Inc., a College of American Pathologists (CAP) certified and Clinical Laboratory Improvement Amendments (CLIA) certified laboratory of 3D Medicines Inc. Genetic alterations were identified, microsatellite instability status (MSI) was assessed, and clinical information including age, gender, and tumor histology were collected. Germline variants were identified by comparing patient's tumor to the matching blood controls. The detected genetic alteration types included single-nucleotide variants (SNVs), insertions/deletions (indels), copy number variations, and gene rearrangements. FGF genes included FGF3, FGF4, FGF6, FGF10, FGF14, FGF19, and FGF23. FGFR genes included FGFR1, FGFR2, FGFR3, and FGFR4. Gene alterations of other cancer signaling pathways, including RTK/RAS pathway,
PI3K pathway, and cell cycle pathway, were also identified[15].

\section{Statistical analysis}

Data were analyzed with GraphPad Prism (version 7.01, GraphPad Software, USA) and R (version 4.3.1, $\mathrm{R}$ Development Core Team). All reported $P$ values were two-sided, and $P<0.05$ was considered statistically significant unless otherwise specified.

\section{Results}

\section{Patient Characteristics}

A total of 12,372 Chinese cancer patients with more than 20 tumor types were included in the study, including 3,557 (29\%) lung cancer, 1,433 (12\%) liver cancer, 1,310 (11\%) colorectal cancer (CRC), 960 (8\%) biliary tract cancer, and $758(6 \%)$ and gastric cancer (GC), etc. (Table 1). The median age was 58 (IQR, 49-66) and 7,440 (60\%) were male patients. NGS was conducted with the FFPE tumor specimens of the patients through surgical resection $(n=7,959,64.3 \%)$, biopsy $(n=3,734,30.2 \%)$, or other approaches $(n=681$, $5.5 \%)$.

Table 1. Clinicopathologic Features of 12,372 Chinese Cancer Cases.

\begin{tabular}{ll}
\hline Characteristics & All patients $(\mathrm{n}=12,372)$ \\
\hline Age, median (IQR) & $58.0(49.0-66.0)$ \\
Sex, $\mathrm{n}(\%)$ & $7440(60)$ \\
Male & $4932(40)$ \\
Female & \\
Histology type, $\mathrm{n}(\%)$ & $3557(29)$ \\
Lung cancer & $1433(12)$ \\
Liver cancer & $1310(11)$ \\
Colorectal cancer & $960(8)$ \\
Biliary Tract cancer & $824(7)$ \\
Gastric cancer & $635(5)$ \\
Pancreas cancer & $548(4)$ \\
Kidney cancer & $544(4)$ \\
Other & $371(3)$ \\
Breast cancer & $369(3)$ \\
Sarcoma & $293(2)$ \\
Ovarian cancer & $275(2)$ \\
Urinary Tract cancer & $295(2)$ \\
Head and Neck carcinoma & $195(2)$ \\
Cervical cancer & $160(1)$ \\
Melanoma & $159(1)$ \\
Esophagus cancer & $136(1)$ \\
Endometrium cancer & $124(1)$ \\
Intestine cancer & $95(1)$ \\
Prostate cancer & $89(1)$ \\
Neuroendocrine cancer &
\end{tabular}

\section{FGF/FGFR Alterations}

Of all patients, FGF/FGFR alterations were observed in 1,943 (15.7\%) patients, including 895 (7.2\%) with alterations in FGF, $862(7.0 \%)$ in FGFR, and $186(1.5 \%)$ in both FGF and FGFR (Figure 1A). Amongst all, there are 1,032 (8.3\%) patients with 
SNVs, 930 (7.5\%) with amplifications, and $116(0.9 \%)$ with fusions in FGF/FGFR genes. The alteration frequencies between FGF and FGFR were mostly comparable in each tumor type. FGF/FGFR mutations were widely occurred across all involving tumor types, with a prevalence ranging from $61.6 \%(98 / 159)$ in esophagus cancer, $52.7 \%(145 / 275)$ in urinary tract cancer, $32.3 \%(83 / 257)$ in head and neck carcinoma to $7.4 \%(7 / 95)$ in prostate cancer, $7.1 \%(45 / 635)$ in pancreatic cancer, and $4.4 \%(24 / 548)$ in kidney cancer (Figure 1A, Table 2). When stratified by the mutational types, the most common pathway-level abnormalities fell in FGFR genes SNVs (635, 5.1\%), FGF genes amplifications $(628,5.1 \%)$, and FGFR genes amplifications $(357,2.9 \%)$. The top frequent gene-level alterations were amplifications in FGF19 (462, 3.7\%), FGF4 (454, 3.7\%), FGF3 (438, 3.5\%), and FGFR1 (220, $1.8 \%)$, followed by SNVs in FGFR2 (222, 1.8\%), FGFR3 (211, 1.7\%), FGFR4 (154, 1.2\%) and FGFR1 (146, 1.2\%). The somatic mutation maps of FGFR1/2/3/4 were shown in Figure 1B.

Table 2. Distribution of patients with FGF/FGFR alterations across different tumor types.

\begin{tabular}{lllllll}
\hline & $\begin{array}{l}\text { FGFR } \\
\text { Amplification }\end{array}$ & $\begin{array}{l}\text { FGFR } \\
\text { SNV }\end{array}$ & $\begin{array}{l}\text { FGFR } \\
\text { Fusion }\end{array}$ & $\begin{array}{l}\text { FGF } \\
\text { Amplificatio } \\
\mathrm{n}\end{array}$ & $\begin{array}{l}\text { FGF } \\
\text { SNV }\end{array}$ & $\begin{array}{l}\text { FGF } \\
\text { Fusion }\end{array}$ \\
\hline $\begin{array}{l}\text { Total } \\
\begin{array}{l}\text { Esophagus } \\
\text { cancer }\end{array}\end{array}$ & $2.9 \%$ & $5.1 \%$ & $0.9 \%$ & $5.1 \%$ & $3.9 \%$ & $0.1 \%$ \\
\hline
\end{tabular}

A

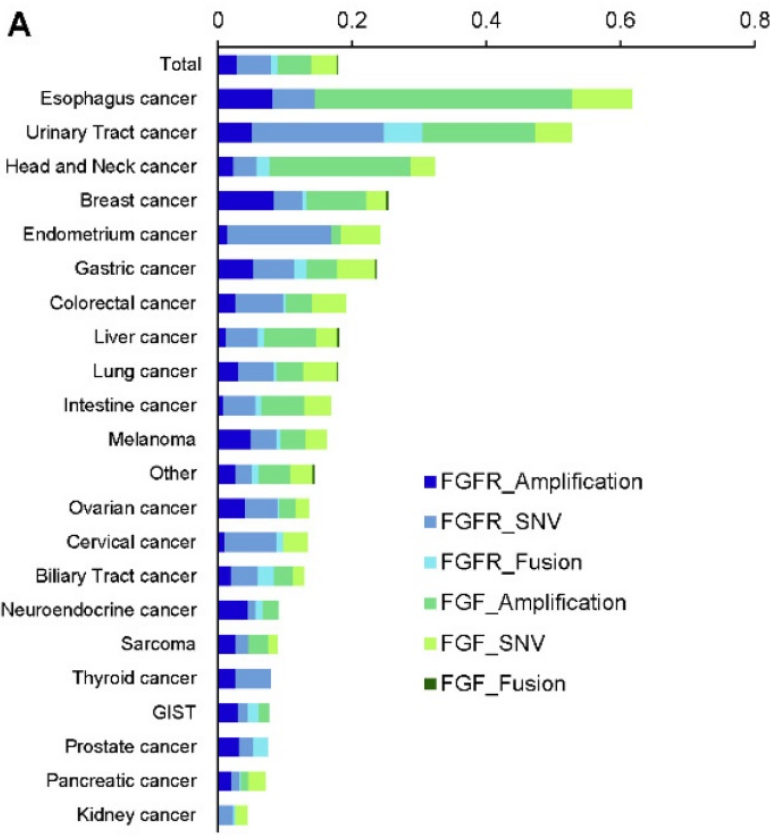

\begin{tabular}{|c|c|c|c|c|c|c|}
\hline & $\begin{array}{l}\text { FGFR } \\
\text { Amplification }\end{array}$ & $\begin{array}{l}\text { FGFR } \\
\text { SNV }\end{array}$ & $\begin{array}{l}\text { FGFR } \\
\text { Fusion }\end{array}$ & $\begin{array}{l}\text { FGF } \\
\text { Amplificatio } \\
n\end{array}$ & $\begin{array}{l}\text { FGF } \\
\text { SNV }\end{array}$ & $\begin{array}{l}\text { FGF } \\
\text { Fusion }\end{array}$ \\
\hline $\begin{array}{l}\text { Urinary Tract } \\
\text { cancer }\end{array}$ & $5.1 \%$ & $19.6 \%$ & $5.8 \%$ & $16.7 \%$ & $5.5 \%$ & $0.0 \%$ \\
\hline $\begin{array}{l}\text { Head and Neck } \\
\text { cancer }\end{array}$ & $2.3 \%$ & $3.5 \%$ & $1.9 \%$ & $21.0 \%$ & $3.5 \%$ & $0.0 \%$ \\
\hline Breast cancer & $8.4 \%$ & $4.3 \%$ & $0.5 \%$ & $8.9 \%$ & $3.0 \%$ & $0.3 \%$ \\
\hline $\begin{array}{l}\text { Endometrium } \\
\text { cancer }\end{array}$ & $1.5 \%$ & $15.4 \%$ & $0.0 \%$ & $1.5 \%$ & $5.9 \%$ & $0.0 \%$ \\
\hline Gastric cancer & $5.3 \%$ & $6.2 \%$ & $1.8 \%$ & $4.5 \%$ & $5.7 \%$ & $0.1 \%$ \\
\hline $\begin{array}{l}\text { Colorectal } \\
\text { cancer }\end{array}$ & $2.6 \%$ & $7.2 \%$ & $0.4 \%$ & $3.9 \%$ & $5.1 \%$ & $0.0 \%$ \\
\hline Liver cancer & $1.2 \%$ & $4.8 \%$ & $1.0 \%$ & $7.7 \%$ & $3.2 \%$ & $0.2 \%$ \\
\hline Lung cancer & $3.1 \%$ & $5.3 \%$ & $0.4 \%$ & $4.0 \%$ & $5.1 \%$ & $0.0 \%$ \\
\hline Intestine cancer & $0.8 \%$ & $4.8 \%$ & $0.8 \%$ & $6.5 \%$ & $4.0 \%$ & $0.0 \%$ \\
\hline Melanoma & $5.0 \%$ & $3.8 \%$ & $0.6 \%$ & $3.8 \%$ & $3.1 \%$ & $0.0 \%$ \\
\hline Other & $2.8 \%$ & $2.4 \%$ & $0.9 \%$ & $4.8 \%$ & $3.3 \%$ & $0.2 \%$ \\
\hline Ovarian cancer & $4.1 \%$ & $4.8 \%$ & $0.3 \%$ & $2.4 \%$ & $2.0 \%$ & $0.0 \%$ \\
\hline Cervical cancer & $1.0 \%$ & $7.7 \%$ & $1.0 \%$ & $0.0 \%$ & $3.6 \%$ & $0.0 \%$ \\
\hline $\begin{array}{l}\text { Biliary Tract } \\
\text { cancer }\end{array}$ & $2.0 \%$ & $4.1 \%$ & $2.4 \%$ & $2.8 \%$ & $1.6 \%$ & $0.0 \%$ \\
\hline $\begin{array}{l}\text { Neuroendocrin } \\
\text { e cancer }\end{array}$ & $4.5 \%$ & $1.1 \%$ & $1.1 \%$ & $2.2 \%$ & $0.0 \%$ & $0.0 \%$ \\
\hline Sarcoma & $2.7 \%$ & $1.9 \%$ & $0.0 \%$ & $3.0 \%$ & $1.4 \%$ & $0.0 \%$ \\
\hline Thyroid cancer & $2.6 \%$ & $5.3 \%$ & $0.0 \%$ & $0.0 \%$ & $0.0 \%$ & $0.0 \%$ \\
\hline GIST & $3.0 \%$ & $1.5 \%$ & $1.5 \%$ & $1.5 \%$ & $0.0 \%$ & $0.0 \%$ \\
\hline Prostate cancer & $3.2 \%$ & $2.1 \%$ & $2.1 \%$ & $0.0 \%$ & $0.0 \%$ & $0.0 \%$ \\
\hline $\begin{array}{l}\text { Pancreatic } \\
\text { cancer }\end{array}$ & $2.0 \%$ & $1.3 \%$ & $0.2 \%$ & $1.1 \%$ & $2.5 \%$ & $0.0 \%$ \\
\hline Kidney cancer & $0.2 \%$ & $2.2 \%$ & $0.4 \%$ & $0.0 \%$ & $1.6 \%$ & $0.0 \%$ \\
\hline
\end{tabular}

\section{Mutual Exclusivity and Co-occurrence}

The relationships between genetic alterations were investigated among FGF/FGFR genes. In brief, alterations in FGF3, FGF4, and FGF19 from 11q13 were mostly concomitant, and were partially
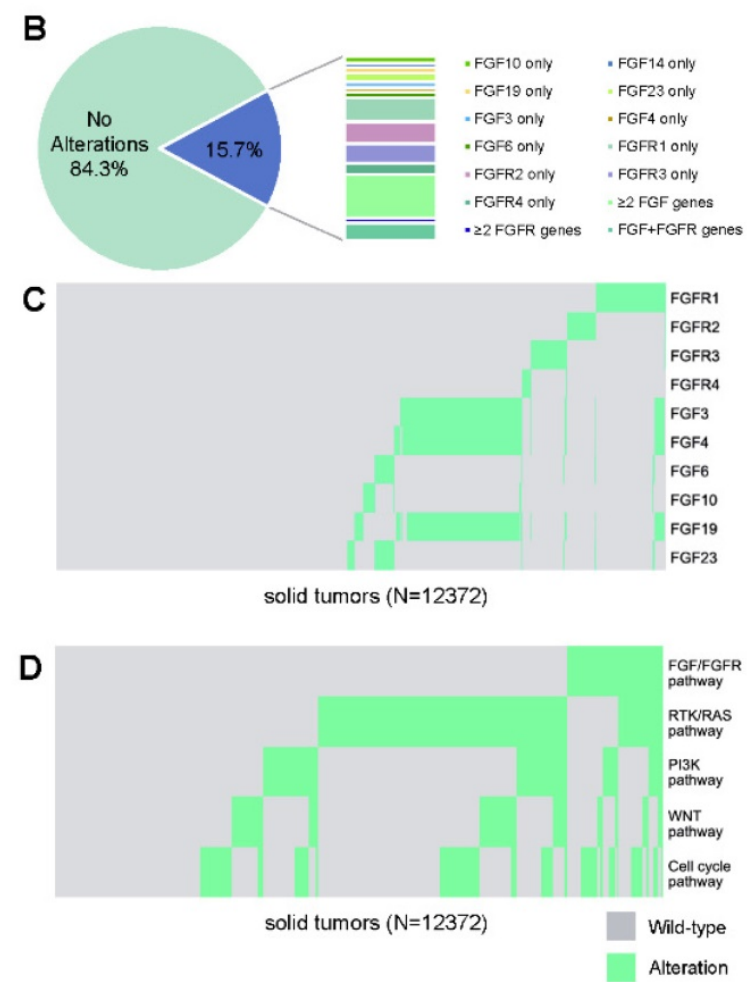

Figure 1. FGFIFGFR genes in cancer patients. (A) Landscape of FGF/FGFR alterations across different cancer types. (B) Distribution of the mutated genes in FGF/FGFR. (C) The mutual exclusivity and co-occurrence relationship with the corresponding significances $(D)$ among the pathway gene alterations. 
co-occurred with FGFR1 alterations (Figure 1C). On the other side, most of the other FGF/FGFR genes were mutually exclusive, for example, the alterations of FGFR1, FGFR2, and FGFR3 ( $\mathrm{p}<0.001)$. The mutual exclusivity and co-occurrence mutational pattern was mostly consistent when constrained to gene amplifications, with the co-occurring FGF3, FGF4, and FGF19 amplifications, and the widely observed mutual exclusivity across the other genes (data not shown). As the top frequent alterations in the overall population, amplifications in FGF3/4/19 (490, 4.0\%) occurred the most in esophagus cancer (60/159, $37.7 \%)$, head and neck carcinoma (54/257, 21.0\%), and urinary tract cancer $(41 / 275,14.9 \%)$, and were the least in pancreatic cancer $(4 / 635,0.6 \%)$, sarcoma $(2 / 369,0.5 \%)$, and ovarian cancer $(1 / 293,0.3 \%)$.

In addition, the occurrence of alterations in different pathways were investigated (Figure 1D). Significantly higher mutational rates of PI3K pathway $(32.0 \%$ vs $20.5 \%, p<0.0001)$, WNT pathway $(21.4 \%$ vs $18.1 \%, p=0.0010)$, and cell cycle pathway $(49.8 \%$ vs $21.9 \%, p<0.0001)$ were observed in patients harboring FGF/FGFR alterations compared to the wild-type patients, while such trend was not found in RTK/RAS pathway $(46.8 \%$ vs $48.7, p=0.1447)$.

\section{Pathway Alterations and Microsatellite Instability}

The status of microsatellite instability (MSI) was available in $10,680(86.3 \%)$ of the patients. In overall, $2.4 \%(258 / 10,680)$ patients showed a high MSI (MSI-H) status. Amongst all, MSI-H was found to be positively associated with the FGF/FGFR alterations $(\mathrm{p}<0.0001)$, with $124(48.1 \%)$ of the MSI-H patients harbored at least one genetic alteration in FGF/FGFR. However, MSI-H was negatively associated with FGF/FGFR amplifications ( $\mathrm{p}=0.0017)$, with only seven $(2.7 \%)$ patients with MSI-H harbored FGF/FGFR gene amplifications.

\section{Discussion}

This is the first study that comprehensively depicting the landscape of FGF/FGFR aberrations in Chinese cancer patients. Our study showed that FGF/FGFR alterations were common across various histologies in Chinese cancer patients.

The recurring oncogenic FGF/FGFR mutations were detected in $15.7 \%$ cancer patients, including $7.2 \%$ with alterations in FGF genes, $7.0 \%$ in FGFR genes, and $1.5 \%$ in both FGF and FGFR genes, which is comparable to the previously reported $7 \%$ of FGFR alterations in the Caucasian cancer population [16]. The distribution of FGFR and FGF alterations were mostly comparable in each of the 20 tumors in the studied cohort, except the dominant FGF alterations observed in esophagus cancer and the dominant FGFR alterations in gynecologic cancer and prostate cancer. For FGFR signaling, the most affected cancer types in the Caucasian cancer population was reported in urothelial (32\%), breast (18\%), and endometrial and squamous cell lung (13\% for each) [16], similar to those in the studied cohort as $30.5 \%$ in urothelial cancer, $16.9 \%$ in endometrium cancer, and $13.2 \%$ in breast cancer. However, the aberrant FGFR signaling was much more common in gastric cancer $(19.4 \%$ vs $6.7 \%)$ and head and neck carcinoma $(15.7 \%$ vs $4.6 \%$ ) in Chinese versus Caucasian patients. With the striking results from the drugs targeting mutant FGF/FGFR in various cancer, non-selective FGF/FGFR inhibitors have been approved for cancer treatment, especially for tumors harboring aberrant FGFR2 or FGFR3 activations [11, 13]. Despite the recently reported disadvantages of a selective inhibitor of FGFR1, 2, and 3 in 22 cholangiocarcinoma patients with FGF/FGFR genetic alterations other than FGFR2 translocation from the interim results of flight-202 study [17], the encouraging efficacy of pan-FGFR inhibitor were reported in patients harboring these alterations with durable response [9]. In addition, the acquired resistance upon FGF/FGFR mutation for various conventional anti-tumor treatment largely impeded the continued treatment. Of note, amplifications in the 11q13 chromosome (including FGF3, FGF4, and FGF19), which was found to be negatively correlated with MSI-H in this study, was reported to be potentially associated with hyperprogression upon immune checkpoint inhibitors as observed in three out of four patients with lung cancer or esophageal adenocarcinoma [18]. Given the different agent sensitivities and efficacies towards various FGF/FGFR genetic alterations, the optimal development of FGF/FGFR axis-target agents required knowledge of the distribution and types of FGF/FGFR aberrations in a variety of cancer types.

There are several limitations in this study. Firstly, given the cross-sectional setting of this study, no treatment or survival data were available for the studied subset, and thus the association between FGF/FGFR alterations with clinical outcome cannot be assessed. Secondly, the cancer subtype was not further identified in the current study, which may lead to potential bias. Collectively, our study has provided valuable clues to the future clinical development, and further investigations are warranted for the clinical application of cancer treatment upon molecular profiling. 


\section{Acknowledgements}

\section{Funding}

The study was supported by a grant from the Education Department of Jiangxi Province (GJJ180008) and a grant from Health Commission of Jiangxi Province (20185145).

\section{Ethics approval}

The study was approved by the Ethics Committee of The First Affiliated Hospital of Nanjing Medical University. All patients provided written informed consent. Patient identity protection was maintained throughout the study.

\section{Consent to participate}

All patients provided written informed consent.

\section{Consent for publication}

All patients signed informed consent regarding publishing their data.

\section{Availability of data and material}

The data that support the findings of this study are available from the corresponding author on reasonable request.

\section{Author Contributions}

Dr. Wei Zuo and Yan He contributed equally to this work and serve as co-first authors. All authors contributed to the study conception and design. Material preparation, data collection and analysis were performed by Wei Zuo, Yan He, Zhengyi Zhao, and Yuzi Zhang. The first draft of the manuscript was written by Zhengyi Zhao and all authors commented on previous versions of the manuscript. All authors read and approved the final manuscript.

\section{Competing Interests}

The authors have declared that no competing interest exists.

\section{References}

[1] Katoh M. Fibroblast growth factor receptors as treatment targets in clinical oncology. Nature reviews Clinical oncology 2019;16:105-22.

[2] Katoh M, Nakagama H. FGF receptors: cancer biology and therapeutics. Medicinal research reviews 2014;34:280-300.

[3] Lemmon MA, Schlessinger J. Cell signaling by receptor tyrosine kinases. Cell 2010;141:1117-34.

[4] Greulich H, Pollock PM. Targeting mutant fibroblast growth factor receptors in cancer. Trends in molecular medicine 2011;17:283-92.

[5] Dienstmann R, Rodon J, Prat A, Perez-Garcia J, Adamo B, Felip E, Cortes J, Iafrate AJ, Nuciforo P, Tabernero J. Genomic aberrations in the FGFR pathway: opportunities for targeted therapies in solid tumors. Annals of oncology : official journal of the European Society for Medical Oncology 2014;25:552-63.

[6] Zhou Y, Wu C, Lu G, Hu Z, Chen Q, Du X. FGF/FGFR signaling pathway involved resistance in various cancer types. Journal of Cancer 2020;11:2000-7.

[7] Roidl A, Berger HJ, Kumar S, Bange J, Knyazev P, Ullrich A. Resistance to chemotherapy is associated with fibroblast growth factor receptor 4 up-regulation. Clinical cancer research : an official journal of the American Association for Cancer Research 2009;15:2058-66.
[8] Lamont FR, Tomlinson DC, Cooper PA, Shnyder SD, Chester JD, Knowles MA. Small molecule FGF receptor inhibitors block FGFR-dependent urothelial carcinoma growth in vitro and in vivo. Br J Cancer 2011;104:75-82.

[9] Dumbrava EI, Alfattal R, Miller VA, Tsimberidou AM. Complete Response to a Fibroblast Growth Factor Receptor Inhibitor in a Patient With Head and Neck Squamous Cell Carcinoma Harboring FGF Amplifications. JCO precision oncology 2018;2.

[10] Touat M, Ileana E, Postel-Vinay S, Andre F, Soria JC. Targeting FGFR Signaling in Cancer. Clinical cancer research : an official journal of the American Association for Cancer Research 2015;21:2684-94.

[11] Loriot Y, Necchi A, Park SH, Garcia-Donas J, Huddart R, Burgess E, Fleming M, Rezazadeh A, Mellado B, Varlamov S, Joshi M, Duran I, Tagawa ST, Zakharia Y, Zhong B, Stuyckens K, Santiago-Walker A, De Porre P, O'Hagan A, Avadhani A, Siefker-Radtke AO, Group BLCS. Erdafitinib in Locally Advanced or Metastatic Urothelial Carcinoma. The New England journal of medicine 2019;381:338-48.

[12] FDA grants accelerated approval to erdafitinib for metastatic urothelial carcinoma. 2019 Acessed.

[13] Abou-Alfa GK, Sahai V, Hollebecque A, Vaccaro G, Melisi D, Al-Rajabi R, Paulson AS, Borad MJ, Gallinson D, Murphy AG, Oh DY, Dotan E, Catenacci DV, Van Cutsem E, Ji T, Lihou CF, Zhen H, Feliz L, Vogel A. Pemigatinib for previously treated, locally advanced or metastatic cholangiocarcinoma: a multicentre, open-label, phase 2 study. The Lancet Oncology 2020.

[14] Su D, Zhang D, Chen K, Lu J, Wu J, Cao X, Ying L, Jin Q, Ye Y, Xie Z, Xiong L, Mao W, Li F. High performance of targeted next generation sequencing on variance detection in clinical tumor specimens in comparison with current conventional methods. Journal of experimental \& clinical cancer research : CR 2017;36:121.

[15] Sanchez-Vega F, Mina M, Armenia J, Chatila WK, Luna A, La KC, Dimitriadoy S, Liu DL, Kantheti HS, Saghafinia S, Chakravarty D, Daian F, Gao Q, Bailey MH, Liang WW, Foltz SM, Shmulevich I, Ding L, Heins Z, Ochoa A, Gross B, Gao J, Zhang H, Kundra R, Kandoth C, Bahceci I, Dervishi L, Dogrusoz U, Zhou W, Shen H, Laird PW, Way GP, Greene CS, Liang H, Xiao Y, Wang C, Iavarone A, Berger AH, Bivona TG, Lazar AJ, Hammer GD, Giordano T, Kwong LN, McArthur G, Huang C, Tward AD, Frederick MJ, McCormick F, Meyerson M, Cancer Genome Atlas Research N, Van Allen EM, Cherniack AD, Ciriello G, Sander C, Schultz N. Oncogenic Signaling Pathways in The Cancer Genome Atlas. Cell 2018;173:321-37 e10.

[16] Helsten T, Elkin S, Arthur E, Tomson BN, Carter J, Kurzrock R. The FGFR Landscape in Cancer: Analysis of 4,853 Tumors by Next-Generation Sequencing. Clinical cancer research : an official journal of the American Association for Cancer Research 2016;22:259-67.

[17] Hollebecque A, Borad M, Sahai V, Catenacci D, Murphy A, Vaccaro G, Paulson A, Oh DY, Féliz L, Lihou C, Zhen H, Abou-Alfa G. 756PInterim results of fight-202, a phase II, open-label, multicenter study of INCB054828 in patients (pts) with previously treated advanced/metastatic or surgically unresectable cholangiocarcinoma (CCA) with/without fibroblast growth factor (FGF)/FGF receptor (FGFR) genetic alterations. Annals of Oncology 2018;29.

[18] Singavi AK, Menon S, Kilari D, Alqwasmi A, Ritch PS, Thomas JP, Martin AL, Oxencis C, Ali S, George B. Predictive biomarkers for hyperprogression (HP) in response to immune checkpoint inhibitors (ICI)-analysis of somatic alternations (SAs). Annals of oncology : official journal of the European Society for Medical Oncology 2017;28(Suppl); Abstract 1140 